\title{
Breast cancer genome analysis in time and space: biomarker development strategy
}

\author{
loannis D Kyrochristos ${ }^{1,2}$, Demosthenes E Ziogas ${ }^{1,3}$, Efstathios G Lykoudis ${ }^{4}$ \& Dimitrios H \\ Roukos ${ }^{*}, 1,2,5$ \\ ${ }^{1}$ Center for Biosystems \& Genome Network Medicine, Ioannina University, loannina, 45110, Greece \\ ${ }^{2}$ Department of Surgery, loannina University Hospital, Ioannina, 45500, Greece \\ ${ }^{3}$ Department of Surgery, 'G Hatzikosta' General Hospital, Ioannina, 45001, Greece \\ ${ }^{4}$ Department of Plastic Surgery, loannina University Hospital, Ioannina, 45500, Greece \\ ${ }^{5}$ Department of Systems Biology, Biomedical Research Foundation of the Academy of Athens (BRFAA), Athens, 11527, Greece \\ *Author for correspondence: Tel.: +30 265100 5572; droukos@uoi.gr
}

First draft submitted: 5 April 2018; Accepted for publication: 6 April 2018; Published online: 6 June 2018

Keywords: biomarkers $\bullet$ breast cancer $\bullet$ genome analysis $\bullet$ prediction $\bullet$ prognosis $\bullet$ therapeutic resistance

Definite evidence on substantial improvement of overall survival rates through screening for the early detection and personalization of therapy has established genetic and molecular biomarkers as standard tools in the management of breast cancer (BC). Spatiotempolar exploration of genomic clones in the primary tumor and circulating cell-free DNA (cfDNA) represents an exciting perspective toward biomarker-directed individualization treatment.

Primary prevention and early detection of $\mathrm{BC}$ represent major steps toward the substantial reductions of advanced disease diagnosis and cancer-related death rates. The establishment of BRCA1 and BRCA2 (BRCA1/2) testing in women with significant family history as an essential tool for risk-reducing surgery has already saved the lives of many women with hereditary breast-ovarian cancer syndrome. Recently, multi-gene panels implementing predisposition genes have been developed for familial BC risk assessment. Furthermore, blood-based early detection is currently under investigation for sporadic cancer [1].

Advances in clinical and translational researches have led to the integration of clinicopathologic and molecular characteristics into BC decision-making algorithms for personalized treatment [2]. However, a substantial proportion of patients, particularly those with advanced stage and triple negative tumors, remains at high risk of relapse and death. The development of robust prognostic and predictive biomarkers for selecting the optimal drug combination is essential for reducing therapeutic resistance and relapse rates in individual patients [3]. Currently, static and dynamic genome analysis attracts major research interest as a rational biomarker development strategy. Revolutionary basic research for understanding dynamic clone evolution in time and space shapes the nextgeneration discoveries toward the individualization of therapy [4].

\section{Clinical \& molecular tools in modern personalized decision-making}

The discovery of germline mutations in BRCA1/2 approximately 25 years ago has revolutionized primary prevention in women with significant family history. Currently, utilization of next-generation sequencing (NGS) technologies for genetic screening with a gene panel of more than a dozen predisposition genes can stratify women into high, moderate- and low-risk for the development of BC, which could determine the primary preventive strategy, including prophylactic surgery, intensive surveillance and mammographic screening.

Primary therapeutic decision-making is based on comprehensive clinicopathologic and molecular patient characteristics, including age, Tumor, Node, Metastasis stage, histological grade as well as ER/PR/HER2 status. Indeed, personalization of treatment on the basis of these clinical and molecular data is crucial for selecting the most effective drug combination, including chemotherapeutic regimens, endocrine therapy and targeted agents [5]. Despite this progress in the individualization of therapy, through applying clinical and molecular tools, a substantial proportion of patients, such as those with late diagnosis and triple-negative status, face increased risk of relapse and death. Understanding spatiotemporal tumor evolution as a predominant cause of therapeutic resistance and recurrence could enable a new generation of genome-based predictive biomarkers. 


\section{Genomic studies on biomarker development}

Beyond the well-established genetic markers already integrated in the management of $\mathrm{BC}$, genome analysis with integrated NGS systems aims to the identification of novel biomarkers with significant clinical implications toward the personalization of treatment. On the one hand, conventional NGS studies with a single-biopsy approach have focused on genomic and transcriptomic analysis with clinical relevance for enhanced early diagnosis, prognostic stratification and prediction of therapeutic response to primary systemic treatment. On the other, dynamic genomic studies exploring tumor evolution in time and space emphasize on the potential prediction of acquired therapeutic resistance and the effect of intratumor heterogeneity (ITH) and tumor subclonality on the fatal hallmarks of cancer, such as progression, metastasis and recurrence.

A series of reports have identified several prognostic biomarkers, both in coding [6-9], as well as in noncoding regulatory regions of cancer-related genes [10,11]. For instance, Ping et al. [6] analyzed 680 BC whole-genomes from The Cancer Genome Atlas [12] and found that several genetic variants are significantly associated with the tumor histologic grade, and subsequently with patient prognosis. Ten genetic factors were identified, including three most prevalent cancer driver events, namely mutations of TP53 and PIK3CA, as well as MYC amplifications. In this cohort, TP53 mutation and MYC amplification were drivers of tumor progression associated with higher histologic grade, while mutations of PIK3CA were found to reduce the oncogenic effects of these alterations, correlating to more favorable histology, suggesting that histologic grade could be a clinical indicator of cancer driver events guiding tailored treatment. Moreover, NGS analysis has identified gene alterations, such as ESR1 mutations, as drivers of metastasis [7] or predictors of therapeutic response [8].

Beyond DNA sequencing at the exome or genome level, analysis of the transcriptome has recently been applied in innovative studies integrating RNA sequencing (RNAseq). Transcriptome sequencing aims at discovering coding and noncoding mutations with functional significance within the tumor ecosystem, as well as developing novel molecular classifications, with potential clinical relevance [13]. On this basis, Gyorffy and colleagues [10] have analyzed the combined findings of whole-exome sequencing, whole-genome sequencing and RNAseq from 930 BCs. Apart from identifying recurrent noncoding mutations, in contrast to a previous large cohort of $560 \mathrm{BC}$ whole genomes [14], the authors detected three coding (CDH1, MAP3K1, TP53) and two noncoding variants (CRTC3, $S T A G 2$ ), which bore prognostic significance in ER-positive/HER2-negative tumors. The functional importance of noncoding variants and particularly promoters had previously been reported by Rheinbay and colleagues, in a large WES cohort that highlighted the correlation between functional mutations leading to FOXA1 overexpression and therapeutic resistance, progression and poor outcomes [11]. These data suggest that the delineation of the complete repertoire of functional coding and noncoding alterations could enable the identification of robust prognostic and predictive biomarkers and warrant extensive further investigations. However, even though there has been data supporting the potential for molecular subtyping of BC based on RNAseq [9], no valid, clinically relevant molecular classification, based on genomic and transcriptomic characteristics, has yet been developed, as in other cancer types.

Moreover, despite the effective modern screening strategy for the early detection of BC, intensive scientific efforts have been put toward the development of a blood-based genetic test to enhance early diagnosis. However, though the concept of a patient-friendly, noninvasive diagnostic test seems highly compelling, a large study by Cohen $e t$ al. [1] combining targeted NGS of cell-free DNA (cfDNA) and protein biomarkers reported a sensitivity as low as 33\% in the diagnosis of early BC. Thus, considering the correlation between tumor burden and cfDNA sequencing accuracy [15], NGS of cfDNA (cfDNA-NGS) at a single time point is currently unsuitable as a biomarker for early diagnosis and further improvements are needed before clinical applicability.

All these exciting data suggest the potentially pivotal role of genome analysis within the future framework of Precision Cancer Medicine. However, the static single-sample design is conceptually unable to predict acquired therapeutic resistance and subsequent tumor progression, metastasis or recurrence, as well as to guide secondary therapeutic decision-making. Consequently, breakthrough-integrated NGS systems have recently been developed, focusing on the identification of dynamic cancer genome evolution, including serial cfDNA-NGS, as well as multiregional (MR-NGS) and single-cell NGS to identify intratumor heterogeneity. As an example, Jansen et al. [16] underlined the potential capacity of repeated cfDNA-NGS over the disease course and during systemic treatment as a predictive and prognostic biomarker, albeit in a very small scale. Additionally, a landmark study by Yates and colleagues [17] has revealed potential utility of spatiotemporal MR-NGS before and after neo-adjuvant therapy in the prediction of secondary therapeutic resistance. To this end, the authors report the identification of dynamically emerging, subclonal, resistance-driving mutations, such as amplifications of CDKG, FGFR2 and MYC, and a 
deletion within RUNX1, concluding that dynamic ITH could be used as a predictive and therapeutic biomarker. At last, several MR-NGS and single-cell NGS studies have identified large genomic changes, namely chromosomal rearrangements and copy number aberrations, as early and stable evolutionary events, as well as bad prognostic factors, associated with resistance and metastasis [18-20].

The results of genomic trials raise rational promises for the future development of robust biomarkers. Nevertheless, the validity of novel discoveries necessitates large-scale analyses, patient stratification according to baseline clinicopathologic and genomic characteristics and strict protocols. Thus, research efforts should focus on the elimination of these drawbacks, in the context of an evidence-based strategy.

\section{Conclusion \& future perspective}

Static single-biopsy genome analysis can lead to the completion of the comprehensive catalog of BC predisposition genes, significantly improving personalized risk assessment and enabling accurate selection of optimal preventive intervention. Moreover, further technical refinement and gene-panel expansion could improve noninvasive early detection [1].

The validity of NGS and methodological recommendations for the valid discovery of biomarkers [21,22], as well as the rapid development and expected further refinement of NGS systems, including serial cfDNA-NGS and single-cell DNA or RNA sequencing, coupled with the definite evidence on genomic and transcriptional heterogeneity [21,23], for the first time shapes the roadmap to the discovery of robust prognostic and predictive biomarkers. The achievement of precise and individualized prediction of intrinsic and acquired resistance was considered, until now, an unrealistic goal. However, understanding whether spatiotemporal evolution [24] or preexistence and stability of a minor subclone within the primary tumor [19], is responsible for therapeutic failure opens new predictive horizons. Identification of the subgroup of patients with stage I and II cancers, at high risk for drug resistance and relapse, could become a reality through MR-NGS or single-cell NGS and the detection of the minor cell subpopulation, comprising a resistant clone within the primary tumor. In late stage III and IV cases, ITH and serial cfDNA-NGS to detect circulating genomic subclones could be used as predictive biomarkers, to target the comprehensive individualized structural mutational landscape with available or novel agents $[4,25]$.

In summary, static single-biopsy NGS analysis and spatiotemporal detection of genomic clones in tumoral and liquid biopsies shape the new generation of prognostic and predictive biomarker development. Particularly dynamic ITH and serial circulating genomic subclone identification appear as the most compelling biomarkers in the adjuvant and neo-adjuvant settings to guide primary and secondary decision on the individualization of therapy. However, until appropriately designed patient-centric genomic trials provide sufficient evidence, the concept of genomic clones and precision cancer medicine remains an ambitious perspective.

\section{Financial \& competing interests disclosure}

The authors have no relevant affiliations or financial involvement with any organization or entity with a financial interest in or financial conflict with the subject matter or materials discussed in the manuscript. This includes employment, consultancies, honoraria, stock ownership or options, expert testimony, grants or patents received or pending, or royalties.

No writing assistance was utilized in the production of this manuscript.

\section{References}

1. Cohen JD, Li L, Wang Y et al. Detection and localization of surgically resectable cancers with a multi-analyte blood test. Science doi:10.1126/science.aar3247 (2018) (Epub ahead of print).

2. National Comprehensive Cancer Network, Inc. (2018). www.nccn.org/

3. Nicolini A, Ferrari P, Duffy MJ. Prognostic and predictive biomarkers in breast cancer: past, present and future. Semin. Cancer Biol. doi:10.1016/j.semcancer.2017.08.010 (2017).

4. Roukos DH. Spatiotemporal diversification of intrapatient genomic clones and early drug development concepts realize the roadmap of precision cancer medicine. Drug Discov. Today 22(8), 1148-1164 (2017).

5. Low SK, Zembutsu H, Nakamura Y. Breast cancer: the translation of big genomic data to cancer precision medicine. Cancer Sci. 109(3), 497-506 (2018).

6. Ping Z, Xia Y, Shen T et al. A microscopic landscape of the invasive breast cancer genome. Sci. Rep. 6, 27545 (2016).

7. Lefebvre C, Bachelot T, Filleron T et al. Mutational profile of metastatic breast cancers: a retrospective analysis. PLoS Med. 13(12), e1002201 (2016). 
8. Ellis MJ, Ding L, Shen D et al. Whole-genome analysis informs breast cancer response to aromatase inhibition. Nature 486(7403), 353-360 (2012).

9. Vu TN, Pramana S, Calza S, Suo C, Lee D, Pawitan Y. Comprehensive landscape of subtype-specific coding and non-coding RNA transcripts in breast cancer. Oncotarget 7(42), 68851-68863 (2016).

10. Gyorffy B, Pongor L, Bottai G et al. An integrative bioinformatics approach reveals coding and non-coding gene variants associated with gene expression profiles and outcome in breast cancer molecular subtypes. Br. J. Cancer doi:10.1038/s41416-018-0030-0 (2018) (Epub ahead of print).

11. Rheinbay E, Parasuraman P, Grimsby J et al. Recurrent and functional regulatory mutations in breast cancer. Nature 547(7661), 55-60 (2017).

12. The Cancer Genome Atlas (2018). https://cancergenome.nih.gov/

13. Bailey P, Chang DK, Nones K et al. Genomic analyses identify molecular subtypes of pancreatic cancer. Nature 531(7592), 47-52 (2016).

14. Nik-Zainal S, Davies H, Staaf J et al. Landscape of somatic mutations in 560 breast cancer whole-genome sequences. Nature 534(7605), 47-54 (2016).

15. Cohen JD, Javed AA, Thoburn C et al. Combined circulating tumor DNA and protein biomarker-based liquid biopsy for the earlier detection of pancreatic cancers. Proc. Natl Acad. Sci. USA 114(38), 10202-10207 (2017).

16. Jansen MP, Martens JW, Helmijr JC et al. Cell-free DNA mutations as biomarkers in breast cancer patients receiving tamoxifen. Oncotarget 7(28), 43412-43418 (2016).

17. Yates LR, Gerstung M, Knappskog S et al. Subclonal diversification of primary breast cancer revealed by multiregion sequencing. Nat. Med. 21(7), 751-759 (2015).

18. Tang $\mathrm{MH}$, Dahlgren $\mathrm{M}$, Brueffer $\mathrm{C}$ et al. Remarkable similarities of chromosomal rearrangements between primary human breast cancers and matched distant metastases as revealed by whole-genome sequencing. Oncotarget 6(35), 37169-37184 (2015).

19. Gao R, Davis A, Mcdonald TO et al. Punctuated copy number evolution and clonal stasis in triple-negative breast cancer. Nat. Genet. 48(10), 1119-1130 (2016).

20. Wang Y, Waters J, Leung ML et al. Clonal evolution in breast cancer revealed by single nucleus genome sequencing. Nature 512(7513), 155-160 (2014).

21. Lawrence MS, Stojanov P, Mermel CH et al. Discovery and saturation analysis of cancer genes across 21 tumour types. Nature 505(7484), 495-501 (2014).

22. Swanton C, Soria JC, Bardelli A et al. Consensus on precision medicine for metastatic cancers: a report from the MAP conference. Ann Oncol. 27(8), 1443-1448 (2016).

23. Tirosh I, Izar B, Prakadan SM et al. Dissecting the multicellular ecosystem of metastatic melanoma by single-cell RNA-seq. Science 352(6282), 189-196 (2016).

24. Eirew P, Steif A, Khattra J et al. Dynamics of genomic clones in breast cancer patient xenografts at single-cell resolution. Nature 518(7539), 422-426 (2015).

25. Murtaza M, Dawson SJ, Tsui DW et al. Non-invasive analysis of acquired resistance to cancer therapy by sequencing of plasma DNA. Nature 497(7447), 108-112 (2013). 